\title{
Hybrid Precoding and Combining Algorithm for Reduced Complexity and Power Consumption Architectures in mmWave Communications
}

\author{
João Pedro Pavia, ${ }^{1,3}$ Nuno Souto, ${ }^{1,3}$ Marco Ribeiro, ${ }^{1,3}$ João Silva ${ }^{1,3}$ and Rui Dinis ${ }^{2,3}$ \\ ${ }^{1}$ Department of Information Science and Technology, ISCTE-University Institute of Lisbon, 1649-026 Lisboa, \\ Portugal. \\ ${ }^{2}$ Faculdade de Ciências e Tecnologias, Universidade Nova de Lisboa, 1099-085 Lisboa, Portugal. \\ ${ }^{3}$ Instituto de Telecomunicações, 1049 - 001 Lisboa, Portugal. \\ Correspondence should be addressed to João Pedro Pavia: Joao_Pedro_Pavia@iscte-iul.pt
}

\begin{abstract}
Future ultrahigh-speed wireless communication systems face difficult challenges due to the fundamental limitations of current technologies working at microwave frequencies. Millimeter-wave (mmWave) technologies can offer large underutilized bandwidths and ease the implementation of large antenna arrays which are required to help overcome the severe signal attenuation that occurs at these frequencies. The high cost and power consumption of a fully digital mmWave precoder and combiner can be reduced by the adoption of hybrid analog/digital designs based on analog phase shifters. The implementation can be simplified even further if each transceiver is connected to an independent antenna subarray and if the phase shifters are replaced by switches, with the possible aid of inverters. In this paper we present an hybrid design algorithm for orthogonal frequency division multiplexing (OFDM) based mmWave MIMO systems, which can cope with different analog architectures that can be adopted at either the RF precoder or combiner, in order to achieve reduced complexity and power consumption. Numerical results show that using the proposed algorithm, all the architectures can achieve good trade-offs between spectral efficiency and simplified implementation.
\end{abstract}

Index Terms-Alternating Direction Method of Multipliers (ADMM), Hybrid Architectures, Switches, mmWave Communications, Massive MIMO, Green Communications.

\section{INTRODUCTION}

Significant advances have been made in the wireless communications field over the last few years to provide higherspeed connections to users. In order to respond to this type of challenge, it is necessary to use advanced signal processing techniques, modulation and equalization schemes, as well as architectures that are efficient in the management of spectral resources. Despite these advances, today's communications systems cannot face the requirements of the new generation of wireless communications. Millimeter-wave-based technologies are one of the most promising candidates for future wireless communication systems. Such interest is justified by the capabilities of these technologies to be able to offer greater underutilized bandwidth as well as to allow a simplified implementation of large antenna arrays, which are crucial to combat the severe signal attenuation and path losses that occurs at these frequencies $[1,2]$. However, it is important to note that large-scale antennas systems must respect the hardware challenges of mmWave circuits. Although hardware components for this band present good capabilities, their high complexity and power consumption impose more constraints on large-antenna systems, precluding the adoption of the same designs used in lower frequencies. This leads to the adoption of a hybrid digital-analog architectures [3, 4]. The hybrid structure splits signal processing into two separate digital and analog parts, which allows the overall circuit complexity and power consumption to be reduced. Several authors have proposed different design algorithms for these schemes [5]-[8]. For example, in [9] the authors showed that algorithms based on alternating minimization allow the achievement of a performance near the optimal fully digital using hybrid precoding solutions with fully-connected and partiallyconnected structures. By using this class of algorithms, it is possible to decouple the problem into two subproblems and to perform an alternated optimization for both the analog and digital precoders. Although, there is a large bandwidth availability in mmWave bands, practical multiple-input multiple output (MIMO) systems will have to operate in frequency selective channels [4]. This fact gives rise to the need to create hybrid schemes for wideband mmWave systems that can handle the fading of multiple paths in this type of channels. Multi-carrier schemes such as orthogonal frequency division multiplexing (OFDM) are often adopted to address such problems $[9,10]$. In this case, a digital precoder/combiner is designed for each subcarrier while a single analog beamformer is applied for the whole OFDM block.

Hybrid architectures often rely on variable phase shifters for the analog beamformers. However, several authors have discussed possible better alternative implementations for the analog precoder/combiner part considering the trade-off between power consumption, hardware complexity, and spectral efficiency. The main solutions are based on switches, inverter-aided switches and antenna selection, which can achieve interesting results depending on the system model and its configurations as can be seen in [11-14]. 


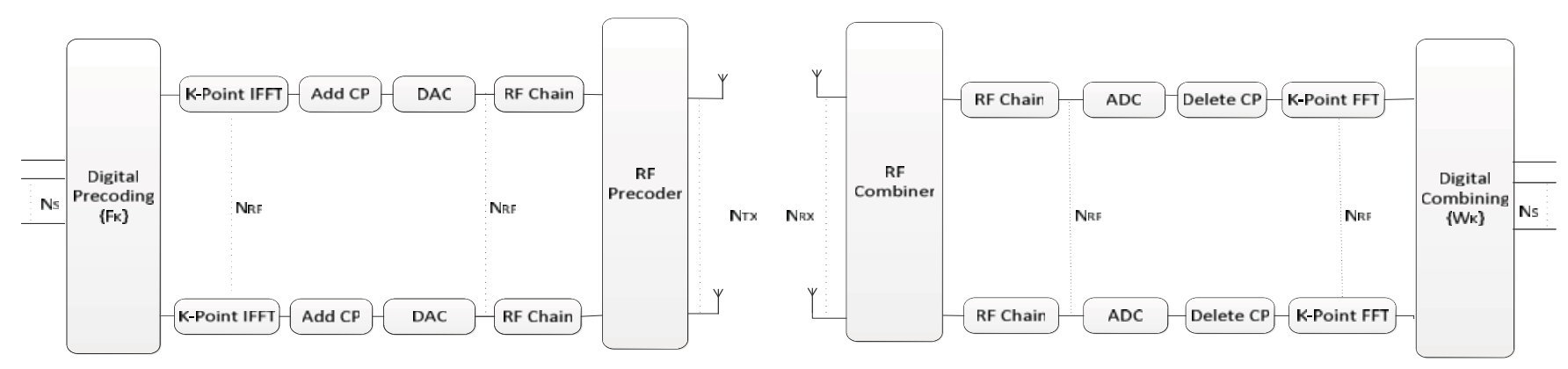

Fig. 1. Hybrid analog/digital precoder and combiner for a OFDM-based mmWave system.

However, most design algorithms are particularly tailored for a specific type of architecture. Motivated by this, we consider the hybrid design for OFDM-based mmWave MIMO systems and develop a general algorithm that can cope with different RF architectures. The different architectures range from quantized phase shifters, to inverter-aided switches, simple switches and antenna selection nodes, all of each can operate in fullyconnected or partially connected configurations and can offer different levels of hardware complexity and power consumption. The proposed algorithm extends the approach that we adopted in [15] for conventional hybrid architectures with unquantized phase shifters and it is also used as a heuristic for providing fast and good quality solutions. Numerical results for the different RF architectures show that, with the presented design approach, good trade-offs between spectral efficiency and hardware implementation complexity can be achieved. The paper is organized as follows: section II presents the system model and problem statement. The proposed algorithm is presented in section III followed by the performance results obtained for each one of the mentioned algorithms in section IV. Finally, the conclusions are outlined in section V.

Notation: Matrices and vectors are denoted by uppercase and lowercase boldface letters, respectively. The superscript $(\cdot)^{H}$ denotes the conjugate transpose of a matrix/vector, $\|\cdot\|_{F}$ is the Frobenius norm, $\mid \cdot$ is the determinant, $\operatorname{tr}(\cdot)$ is the trace of a matrix, $\varnothing$ is the Hadamard product and $\mathbf{I}_{n}$ is the $n \times n$ identity matrix.

\section{System Model AND Problem Statement}

Let us consider a OFDM-based mmWave hybrid MIMO system as shown in Fig.1, where the base station (BS) is equipped with $N_{t x}$ antennas and transmits $N_{s}$ data streams to a receiver containing $N_{r x}$ antennas. The number of RF chains, $N_{R F}$, is assumed to be the same at the transmitter and receiver and satisfies $N_{s} \leq N_{R F} \leq \min \left(N_{t x}, N_{r x}\right)$. Although our approach is independent of a specific channel, we will adopt a wideband mmWave model with $N_{c l}$ scattering clusters, each with $N_{\text {ray }}$ propagations paths. Assuming the operation with blocks of $K$ subcarriers, the frequency domain channel matrix at the $k^{\text {th }}$ subcarrier can be written as

$$
\mathbf{H}[k]=\gamma \sum_{i=1}^{N_{c l}} \sum_{l=1}^{N_{r a y}} \alpha_{i, l} \mathbf{a}_{r}\left(\phi_{i, l}^{r}, \theta_{i, l}^{r}\right) \mathbf{a}_{t}\left(\phi_{i, l}^{t}, \theta_{i, l}^{t}\right)^{H} e^{-j 2 \pi(i-1) k / K}
$$

where $\alpha_{i, l}$ is the complex gain of the $l^{\text {th }}$ ray from cluster $i$ and $\gamma$ is a normalizing factor such that $\mathrm{E}\left[\|\mathbf{H}\|_{F}^{2}\right]=N_{t x} N_{r x}$. Vectors $\mathbf{a}_{t}\left(\phi_{i, l}^{t}, \theta_{i, l}^{t}\right)$ and $\mathbf{a}_{r}\left(\phi_{i, l}^{r}, \theta_{i, l}^{r}\right)$ represent the transmit and receive antenna array responses at the azimuth and elevation angles of $\left(\phi_{i, l}^{t}, \theta_{i, l}^{t}\right)$ and $\left(\phi_{i, l}^{r}, \theta_{i, l}^{r}\right)$, respectively. A different digital baseband precoding $\mathbf{F}_{\mathrm{BB}}[k]$ is applied before the IFFT for each subcarrier, $k$, while a common analog beamformer $\mathbf{F}_{\mathrm{RF}}$ is applied in the time domain to the whole block, as shown in Fig. 1. At the receiver, a similar approach is used, with the RF combiner, $\mathbf{W}_{\mathrm{RF}}$, applied to the whole block and the baseband combiner, $\mathbf{W}_{\mathrm{Bв}}[k]$, applied after the FFT. The resulting signal at subcarrier $k$ after the combiner can be expressed as

$\mathbf{y}[k]=\sqrt{\varepsilon} \mathbf{W}_{\mathrm{BB}}^{H}[k] \mathbf{W}_{\mathrm{RF}}^{H} \mathbf{H}[k] \mathbf{F}_{\mathrm{RF}} \mathbf{F}_{\mathrm{BB}}[k] \mathbf{s}[k]+\mathbf{W}_{\mathrm{BB}}^{H}[k] \mathbf{W}_{\mathrm{RF}}^{H} \mathbf{n}[k]$

where $\varepsilon$ is the average received power, $\mathbf{H}[k] \in \mathbb{C}^{N_{r x} \times N_{k x}}$ is the respective channel matrix (assumed to be perfectly known at the transmitter and receiver) and $\mathbf{n}[k] \in \mathbb{C}^{N_{r x} \times 1}$ contains independent zero-mean circularly symmetric Gaussian noise samples with covariance $\sigma_{n}^{2} \mathbf{I}_{N_{r x}}$. For ease of exposition, we limit most of the presentation to the hybrid precoder design, even though it can also be directly applied to the hybrid combiner design (as is done for the numerical results). Let us define $\mathbf{F}_{\text {opt }}[k]$ as the unconstrained optimum precoding matrix, formed using the first $N_{s}$ columns of matrix $\mathbf{V}_{k}$ which is obtained from the singular value decomposition $\mathbf{H}[k]=\mathbf{U}_{k} \boldsymbol{\Sigma}_{k} \mathbf{V}_{k}{ }^{H}$ of the channel matrix ( $\boldsymbol{\Sigma}_{k}$ is a diagonal matrix with the singular values in decreasing order). Similarly to $[9,15]$ we can approximate the hybrid design problem as a matrix factorization problem applied separately to the precoder and combiner sides. Therefore, the hybrid precoding matrices that maximize the data rate are obtained as the solutions of the following nonconvex optimization problem

$$
\begin{aligned}
& \min _{\mathbf{F}_{\mathrm{RF}}, \mathbf{F}_{\mathrm{BB}}[k]} \sum_{k=0}^{K-1}\left\|\mathbf{F}_{\text {opt }}[k]-\mathbf{F}_{\mathrm{RF}} \mathbf{F}_{\mathrm{BB}}[k]\right\|_{F}^{2} \\
& \text { subject to } \mathbf{F}_{\mathrm{RF}} \in \mathcal{C}_{N_{t x} \times N_{R F}} \\
& \left\|\mathbf{F}_{\mathrm{RF}} \mathbf{F}_{\mathrm{BB}}[k]\right\|_{F}^{2}=N_{s}, k=0, \ldots, K-1,
\end{aligned}
$$

where $\mathcal{C}_{N_{K} \times N_{R F}}$ is the set representing the constraints imposed by the specific RF architecture. 


\section{GenERAl Hybrid PreCODING/COMBINING AlgORITHM}

\section{A. Main Algorithm}

In order to derive a hybrid precoder/design algorithm that can cope with the different RF architectures we can integrate the RF constraint directly into the objective function of the optimization problem. This can be accomplished through the addition of an auxiliary variable, $\mathbf{R}$, combined with the use of the indicator function for set $\mathcal{C}_{N_{t} \times N_{R F}}$. This function is defined as $I_{\mathcal{C}_{N_{L} \times N_{R F}}}(\mathbf{R})$, returning 0 if $\mathbf{R} \in \mathcal{C}_{N_{k x} \times N_{R F}}$ and $+\infty$ otherwise.

The optimization problem can then be rewritten as

$$
\begin{aligned}
& \min _{\mathbf{F}_{\mathrm{RF}}, \mathbf{F}_{\mathrm{BB}}[k]} \sum_{k=0}^{K-1}\left\|\mathbf{F}_{o p t}[k]-\mathbf{F}_{\mathrm{RF}} \mathbf{F}_{\mathrm{BB}}[k]\right\|_{F}^{2}+I_{\mathcal{C}_{N_{k} \times N_{R F}}}(\mathbf{R}) \\
& \quad \text { subject to } \mathbf{F}_{R F}-\mathbf{R}=0
\end{aligned}
$$

In this formulation, the total power constraint (5) is not included since it is not required for the combiner and, in the case of the precoder, it can be handled at the end of the main algorithm (similarly to what is done in [6] and [11]). With the use of the auxiliary variable $\mathbf{R}$ and indicator function the objective function becomes separable which allows us to obtain an iterative algorithm comprising the sequence of steps shown in Table I when we apply ADMM [15]. In Table I, $Q$ denotes the maximum number of iterations. Step 10 ensures that the total power constraint is satisfied and is only applied in the case of the precoder. In Step $5, \prod_{\mathcal{C}_{a \times b}}(\cdot)$ denotes the projection onto set $\mathcal{C}_{a \times b}$. This projection is the only step that is specific to each different architecture as will be explained in the next subsection. Regarding the complexity, steps 3 and 4 (updates of $\mathbf{F}_{R F}{ }^{(t+1)}$ and $\mathbf{F}_{B B}{ }^{(t+1)}$ ) correspond to the main contributions and result in a total complexity order for the algorithm of $\mathcal{O}\left(Q\left(N_{s} N_{R F} N_{t x}+N_{R F}^{2} N_{t x}\right)\right)$

\section{TABLE I}

GENERAL ITERATIVE HYBRID DESIGN ALGORITHM

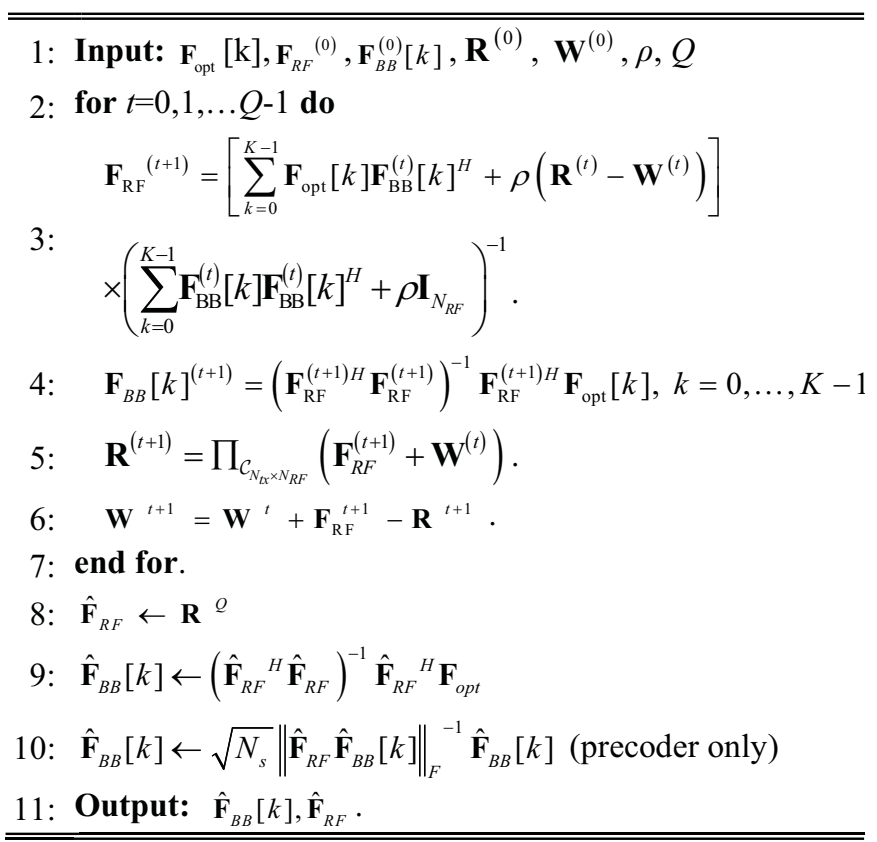

The algorithm in Table I is presented in the general form where one assumes that the architecture is fully connected. For the case of a partially connected structure where each RF chain is linked to a subarray of $N_{t x / r x} / N_{R F}$ antennas, we can simplify it. First, we note that the RF precoder can be expressed as

$$
\mathbf{F}_{\mathrm{RF}}=\left[\begin{array}{cccc}
\mathbf{f}^{R F, 1} & 0 & \cdots & 0 \\
0 & & & \vdots \\
\vdots & & \ddots & 0 \\
0 & \cdots & 0 & \mathbf{f}^{R F, N_{R F}}
\end{array}\right]
$$

where $\mathbf{f}^{R F, i}=\left[f_{1}^{R F, i}, \ldots, f_{\frac{N_{t x}}{N_{R F}}}^{R F, i}\right]^{T} \in \mathcal{C}_{N_{t x} / N_{R F} \times 1}$ with $i=1, \ldots, N_{R F}$.

The optimization problem (3)-(5) can then be formulated as

$$
\begin{gathered}
\min _{\mathbf{f}^{R F, i}, \mathbf{F}_{\mathrm{BB}}[k]} \sum_{k=0}^{K-1} \sum_{j=1}^{N_{t x}} \| \mathbf{F}_{o p t_{j,:}}[k]-f_{\bmod \left(j-1, \frac{N_{t x}}{N_{R F}}\right)+1}^{\left.R F, \frac{j-1}{N_{t x} / N_{R F}}\right]^{+1}} \mathbf{F}_{\mathrm{BB}}\left[\frac{j-1}{N_{t x} / N_{R F}} \mid,[k] \|_{2}^{2}\right. \\
+\sum_{i=1}^{N_{R F}} I_{\mathcal{C}_{N_{t x} \times 1}}\left(\mathbf{r}^{i}\right)
\end{gathered}
$$$$
\text { subject to } \mathbf{f}^{R F, i}-\mathbf{r}^{i}=0, i=1, \ldots, N_{R F}
$$

Applying ADMM to this formulation results in the following modified expressions for steps 3 to 6 in Table I

$$
\begin{aligned}
& \left(f_{j}^{R F, i}\right)^{(t+1)}=\frac{\sum_{k=0}^{K-1} \mathbf{F}_{\mathrm{opt}_{(i-1)} N_{k x}}[k] \mathbf{F}_{\mathrm{BB}_{i, i}}^{(t)}[k]^{H}+\rho\left(r_{j}^{i^{(t)}}-w_{j}^{i^{(t)}}\right)}{\sum_{k=0}^{K-1}\left\|\mathbf{F}_{\mathrm{BB}_{i, j}}^{(t)}[k]\right\|^{2}+\rho}, \\
& i=1, \ldots, N_{R F}, j=1, \ldots, \frac{N_{t x}}{N_{R F}} \\
& \mathbf{F}_{B B_{i,}}{ }^{(t+1)}[k]=\left\|\mathbf{f}^{R F, i^{(t)}}\right\|^{-2}\left(\mathbf{f}^{R F, i^{(t 1)}}\right)^{H} \mathbf{F}_{\mathrm{opt}_{(i-1) \frac{N_{K}}{N_{R F}}}\left(i \frac{N_{N K}}{N_{R F}},\right.}[k], \\
& i=1, \ldots, N_{R F}(12) \\
& \mathbf{r}^{i^{(t 1)}}=\prod_{\mathcal{C}_{N_{t x} / N_{R F} \times 1}}\left(\mathbf{f}^{R F, i^{(t 1)}}+\mathbf{w}^{i^{(t)}}\right), i=1, \ldots, N_{R F} \\
& \mathbf{W}^{i^{(t+1)}}=\mathbf{w}^{i^{(t)}}+\mathbf{f}^{R F, i^{(t+1)}}-\mathbf{r}^{i^{(t+1)}}, i=1, \ldots, N_{R F} .
\end{aligned}
$$

Note that, unlike the general algorithm, these expressions operate with vectors instead of matrices. Regarding the initialization and termination of the algorithm, the same approach described in [15] can be adopted.

\section{B. RF Architectures}

The projection in line 5 of Table I and in (12) can be easily implemented according to the specific analog beamformer. In the following we will consider different architectures that can be adopted at either the RF precoder or RF combiner for achieving reduced complexity and power consumption implementations. 
1) Unquantized Phase Shifters (UPS)

If we assume the use of infinite resolution phase shifter, the $\mathrm{RF}$ constraint set is given by

$$
\mathcal{C}_{a \times b}=\left\{\mathbf{X} \in \mathbb{C}^{a \times b}:\left|X_{i, j}\right|=1 / \sqrt{a}\right\},
$$

and the projection in line 5 of Table I and in (12) can be performed using

$$
\mathbf{R}^{(t+1)}=\left(\mathbf{F}_{R F}^{(t+1)}+\mathbf{W}^{(t)}\right) \varnothing\left|\mathbf{F}_{R F}^{(t+1)}+\mathbf{W}^{(t)}\right|,
$$

where I. represents the elementwise absolute value.

\section{2) Quantized Phase Shifters (QPS)}

Considering the more realistic scenario where digitally controlled phase shifters with $N_{b}$ bits are used, which allow the

selection of $2^{N_{b}}$ different quantized phases, then the RF constraint set becomes

$$
\mathcal{C}_{a \times b}=\left\{\mathbf{X} \in \mathbb{C}^{a \times b}: X_{i, j}=1 / \sqrt{a} e^{2 \pi k i / 2^{N_{b}}}, k=0, \ldots, 2^{N_{b}}-1\right\} .
$$

In this case, we can implement the projection in line 5 of Table I and in (12) as the following element-wise quantization

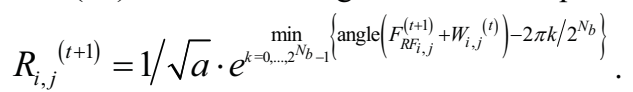

\section{3) Switches and inverters (SI)}

In the previous architecture, if we assume that $N_{b}=1$, then each variable phase shifter can be replaced by a pair of switched lines, where one also includes an inverter. The constraint set reduces to

$$
\mathcal{C}_{a \times b}=\left\{\mathbf{X} \in \mathbb{R}^{a \times b}: X_{i, j}= \pm 1 / \sqrt{a}\right\},
$$

and the projection simplifies to

$$
R_{i, j}{ }^{(t+1)}=1 / \sqrt{a} \cdot \operatorname{sign}\left(\operatorname{Re}\left[F_{R F_{i, j}}^{(t+1)}+W_{i, j}{ }^{(t)}\right]\right) .
$$

\section{4) Switches (Swi)}

As an alternative to the previous simplification we can simply replace each of the variable phase shifters by a switch. This results in a network of switches connecting each RF chain to the antennas. The RF constraint set can be expressed as

$$
\mathcal{C}_{a \times b}=\left\{\mathbf{X} \in \mathbb{R}^{a \times b}: X_{i, j}=0 \vee X_{i, j}=1 / \sqrt{a}\right\}
$$

and the projection can be implemented elementwise as

$$
R_{i, j}{ }^{(t+1)}=1 / \sqrt{a} \cdot\left[1 / 2+1 / 2 \cdot \operatorname{sign}\left(2 \operatorname{Re}\left[F_{R F_{i j}}^{(t+1)}+W_{i, j}{ }^{(t)}\right]-1 / \sqrt{a}\right)\right] .
$$

\section{5) Antenna Selection $(A S)$}

The simplest of all the architecture that we consider here corresponds to the case where each RF chain can be only connected to a single antenna (and vice-versa). The corresponding constraint set comprises matrix with only one nonzero element per column and per row, i.e.,

$$
\mathcal{C}_{a \times b}=\left\{\mathbf{X} \in \mathbb{R}^{a \times b}: X_{i, j}=0 \vee X_{i, j}=1 / \sqrt{a},\left\|\mathbf{X}_{i,}\right\|_{0}=1,\left\|\mathbf{X}_{i, j}\right\|_{0}=1\right\} .
$$

In this definition \|\|$_{0}$ is the 0 -norm, i.e., the cardinality of a vector. Defining $\mathbf{X}=\mathbf{F}_{R F}^{(t+1)}+\mathbf{W}^{(t)}$, the projection can be approximately implemented by setting all the elements in $\mathbf{R}$ as 0 except for $R_{t_{j}, j}{ }^{(t+1)}=1 / \sqrt{a}$, where $t_{j}$ is the row position with the highest real component in column $j$ :

$$
t_{j}=\underset{i=1, \ldots, N_{\text {Nx }}}{\arg \max }\left\{\operatorname{Real}\left[X_{i, j}\right]\right\} .
$$

This computation is performed for all columns $j=1, \ldots, N_{R F}$, sorted by descending order in terms of highest real components and noting that the same row cannot be repeated.

\section{NUMERICAL RESULTS}

In this section, the performance of the proposed ADMM algorithm will be evaluated for OFDM mmWave systems with different analog architectures. We assume that $N_{s}=4$ data streams are sent from the BS over $K=128$ subcarriers, with $N_{t x}=100$ and $N_{r x}=36$, while both are equipped with uniform planar arrays (UPAs). The number of RF chains at the transmitter and at the receiver is $N_{R F}=4$. The channel parameters are given by $N_{c l, k}=1$ and $N_{\text {ray }, k}=8$. The angles of departure and arrival were selected according to a Gaussian distribution with uniformly distributed mean angles in $[0,2 \pi]$ and an angular spread of 10 degrees. A separation of half wavelength distance between the antenna elements in UPAs was considered and all simulation results were computed with 1000 independent Monte Carlo runs.

Fig.2 shows the spectral efficiency versus the signal to noise ratio (SNR), defined as $S N R=\varepsilon / \sigma_{n}^{2}$, achieved by the proposed algorithm considering the effect of quantization. The unquantized ADMM algorithm and Orthogonal Matching Pursuit (OMP) based sparse method [6] are included as benchmarks. It is assumed that, given the unconstrained data rate maximizing precoder/combiner matrices, each algorithm is applied for both precoding and combiner design. As can be seen in Fig. 2, as the number of quantization bits, $N_{b}$, increases the spectral efficiency improves and with only 4 quantization bits, the performance becomes almost the same as the unquantized scheme.

Fig.3 shows the spectral efficiency versus SNR for different receiver architectures. In these numerical results we consider the use of the reduced complexity architectures at the combiner only, since we assume the typical scenario where the mobile station (receiver) has more demanding power consumption constraints when compared to the base station (transmitter). Therefore, the precoder is fixed with a fully connected architecture employing quantized phase shifters $\left(N_{b}=4\right)$. From these results, we can observe that, as expected, the architectures based on quantized phase shifters present a greater spectral efficiency when compared to all the other designs. 


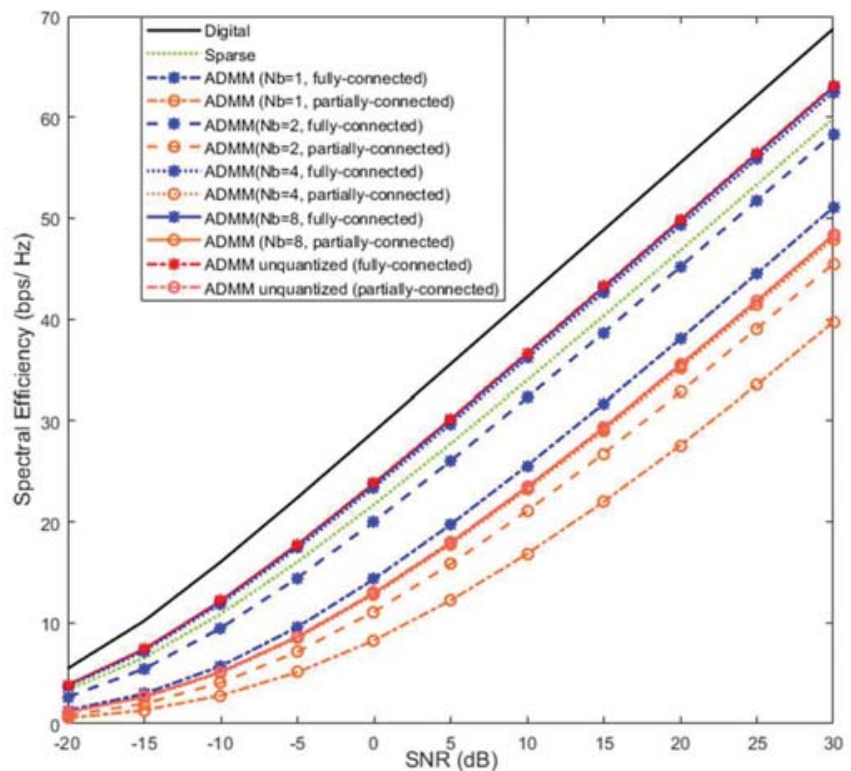

Fig. 2. Spectral efficiency versus SNR considering different levels of quantization on the ADMM precoder/combiner.

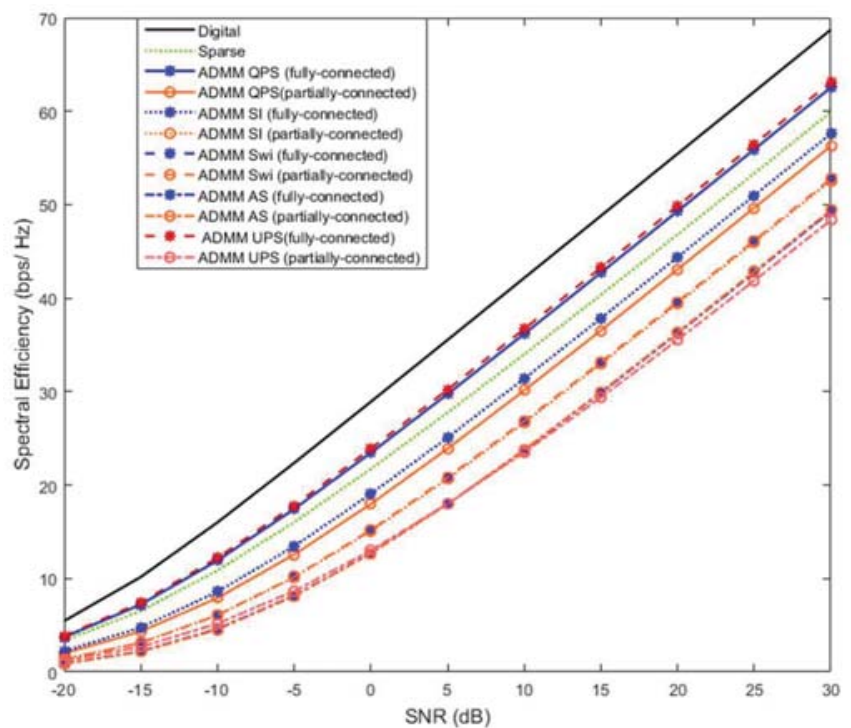

Fig. 3. Spectral efficiency versus SNR achieved by different architectures at the combiner.

We also found that the second most efficient architecture is based on switches and inverters and the last ones are the ones, that are based on switches and antenna selection, respectively. Moreover, there does not seem to be much difference in using "fully-connected" or "partially-connected" in these combiner's architectures.

\section{CONCLUSIONS}

In this paper, we proposed an hybrid design algorithm for spatial multiplexing in OFDM-based mmWave systems operating in frequency selective channels. Our proposed algorithm is obtained through ADMM which, allows us to split a properly formulated design problem into a sequence of smaller subproblems with straightforward solutions. This enables the analog design part to be reduced to a simple projection operation resulting in a flexible algorithm that can easily cope with different architectures, namely variable phase shifter, switches and inverters deployed in fully-connected or partially-connected structures. Numerical results demonstrate that good trade-offs between spectral efficiency and hardware implementation complexity can be achieved with the proposed approach.

\section{ACKNOWLEDGMENT}

This work was partially supported by the FCT - Fundação para a Ciência e Tecnologia and Instituto de Telecomunicações under projects UIDB/EEA/50008/2020, TUBITAK/0002/2014 and MASSIVE5G (SAICT-45-2017-02).

\section{REFERENCES}

[1] X. Ge, S. Tu, G. Mao, C. X. Wang, and T. Han, "5G Ultra-Dense Cellular Networks," IEEE Wireless Commun. Mag., vol. 23, no. 1, pp. 72-79, Feb. 2016.

[2] T. S. Rappaport, S. Sun, R. Mayzus, H. Zhao, Y. Azar, K. Wang, G. N. Wong, J. K. Schulz, M. Samimi and F. Gutierrez, "Millimeter wave mobile communications for $5 \mathrm{G}$ cellular: It will work!" IEEE Access, vol. 1, pp. 335-349, May 2013.

[3] R. Baldemai, T. Irnich, K. Balachandran, E. Dahlman, G. Mildh, Y. Selén, S. Parkvall, M. Meyer, and A. Osseiran, "Ultra-dense networks in millimeter-wave frequencies," IEEE Commun. Mag., vol. 53, no. 1, pp. 202-208, Jan. 2015.

[4] K. Guan, G. Li, T. Kurner, A. F. Molisch, B. Peng, R. He, B. Hui, J. Kim, and Zhangdui Zhong, "On Millimeter Wave and THz Mobile Radio Channel for Smart Rail Mobility," IEEE Trans. Vehicular Techn., vol. 66, no. 7 , pp. 5658-5674, July 2017

[5] A. Alkhateeb, J. Mo, N. Gonzáles-Prelcic, and R. W. Heath, Jr., "MIMO precoding and combining solutions for millimeter-wave systems," IEEE Commun. Mag., vol. 52, no. 12, pp. 122-131, Dec. 2014.

[6] F. Sohrabi and W. Yu, "Hybrid digital and analog beamforming design for large-scale MIMO systems," in Proc. IEEE Int. Conf. Acoust., Speech Signal Process., pp. 2929-2933, Apr. 2015.

[7] C. E. Chen, "An iterative hybrid transceiver design algorithm for millimeter wave MIMO systems," IEEE Wireless Communications Letters, vol. 4, no. 3, pp. 285-288, June 2015.

[8] C. Rusu, R. Mendez-Rial, N. Gonzalez-Prelcic, and R. Heath, "Low complexity hybrid precoding strategies for millimeter wave communication systems," IEEE Trans. Wireless Commun., vol. 15, no. 12, pp. 8380-8393, Dec. 2016.

[9] X. Yu, J. Shen, J. Zhang and K. B. Letaief, "Alternating Minimization Algorithms for Hybrid Precoding in Millimeter Wave MIMO Systems," in IEEE Journal of Selected Topics in Signal Processing, vol. 10, no. 3, pp. 485-500, 2016.

[10] J. Lee and Y. H. Lee, "AF relaying for millimeter wave communication systems with hybrid RF/baseband MIMO processing," 2014 IEEE International Conference on Communications (ICC), Sydney, NSW, pp. 5838-5842, 2014.

[11] R. Mendez-Rial, C. Rusu, N. Gonzalez-Prelcic, A. Alkhateeb and R. Heath, "Hybrid MIMO Architectures for Millimeter Wave Communications: Phase Shifters or Switches?", IEEE Access, vol. 4, pp. 247-267, 2016.

[12] X. Gao, L. Dai, Y. Sun, S. Han and I. Chih-Lin, "Machine learning inspired energy-efficient hybrid precoding for mmWave massive MIMO systems", 2017 IEEE International Conference on Communications (ICC), 2017

[13] S. Payami, M. Ghoraishi, M. Dianati and M. Sellathurai, "Hybrid Beamforming with a Reduced Number of Phase Shifters for Massive MIMO Systems", IEEE Transactions on Vehicular Technology, vol. 67, no. 6, pp. 4843-4851, 2018.

[14] M. Tian, J. Zhang, Y. Zhao, L. Yuan, J. Yang and G. Gui, "Switch and Inverter Based Hybrid Precoding Algorithm for mmWave Massive MIMO System: Analysis on Sum-Rate and Energy-Efficiency", IEEE Access, vol. 7, pp. 49448-49455, 2019.

[15] N. Souto, J. Silva, J. Pavia and M. Ribeiro, "An alternating direction algorithm for hybrid precoding and combining in millimeter wave MIMO systems", Physical Communication, vol. 34, pp. 165-173, 2019. 